\title{
The microbiome of the maxillary sinus and middle nasal meatus in chronic rhinosinusitis*
}

\author{
O.A. Ivanchenko', S.A. Karpishchenko², R.S. Kozlov³, O.I. Krechikova3, \\ I.V. Otvagin ${ }^{4}$, O.N. Sopko², G.Z. Piskunov, A.S. Lopatin ${ }^{6}$ \\ Polyclinic No.121, Moscow Healthcare Department, Moscow, Russia \\ 2Ear, Nose and Throat Clinic and Department, Pavlov First State Medical University, Saint Petersburg, Russia \\ ${ }^{3}$ Institute of Antimicrobial Chemotherapy, State Medical Academy, Smolensk, Russia \\ ${ }^{4}$ Otolaryngology Clinic, State Medical Academy, Smolensk, Russia \\ Otolaryngology Clinic, Russian Medical Academy of Postgraduate Education, Moscow, Russia \\ ${ }^{6}$ Polyclinic No.1, Medical Department, Business Administration of the President of the Russian Federation, Moscow, Russia
}

Rhinology 54: 68-74, 2016

DOI:10.4193/Rhino15.018

*Received for publication:

January 23, 2015

Accepted: August 20, 2015

\begin{abstract}
Aim: This multicenter study was focused on the identification of the microorganisms inhabiting the maxillary sinus and middle nasal meatus in chronic rhinosinusitis.
\end{abstract}

Methodology: 112 middle meatus swabs and 112 maxillary sinus aspirates from 103 patients were available for culture.

Results: A total of 244 strains of microorganisms representing more than 50 families were identified in the maxillary sinus and middle nasal meatus (164 and 80, respectively). These included 154 (63.0\%) strains of aerobic bacteria from 32 species and 90 (37.0\%) strains of anaerobic bacteria from 23 species. Aerobes were more common than anaerobes in both the nasal cavity $(78.7 \%$ vs. $21.3 \%$ ) and in the maxillary sinus (55.2\% vs. $44.8 \%$ ). Species of Streptococci (28.8\%) and Prevotella (17.8\%) were the most common findings in the maxillary sinus aspirates. S. pneumonia, H. influenza, and S. aureus were relatively rare, and found in only $6.7 \%$, $5.4 \%$, and $8.9 \%$ of the samples, respectively.

Conclusions: The results obtained suggest that common upper airway pathogens do not play a major role in the pathogenesis of chronic rhinosinusitis. The microbiome of inflamed sinonasal mucosa is extremely diverse and involves exotic species of bacteria that, to date, have not been considered as potential inhabitants of the paranasal sinuses.

Key words: chronic rhinosinusitis, microbiology, culture dependent techniques, maxillary sinus, middle nasal meatus

\section{Introduction}

Chronic rhinosinusitis (CRS) is, in fact, a cluster of heterogeneous disorders that significantly impacts the patients' quality of life and personal health care expenditure. According to the European Position Paper on Rhinosinusitis and Nasal Polyp (EPOS, 2012), the prevalence of CRS is increasing annually. Recent data have demonstrated that CRS affects approximately $5-15 \%$ of the population in both Europe and the USA ${ }^{(1-4)}$. Currently, CRS is diagnosed when specific sinonasal symptoms last for 12 weeks or more. Modern classification divides CRS patients into those who have nasal polyps (CRSwNP) and those who do not (CRSsNP) ${ }^{(4)}$. Despite major progress that has been made in this field, we still do not have a clear understanding of the pathogenic mechanisms and triggering factors involved in this disease. In recent years, the role of bacteria in the pathogenesis of CRS has been the subject of intense debate, and CRS microbiology has been studied extensively. Bacterial associations found in the paranasal sinuses and nasal cavity in CRS patients contain a large variety of aerobic, facultative anaerobic and anaerobic (both pathogenic and commensal) organisms: Peptostreptococcus spp., Bacteroides spp., Veillonella spp., Prevotella spp., Fusobacterium spp., Corynebacterium spp., Staphylococcus spp., Streptococcus spp., Haemophilus spp., Helicobacter pylori and other Gram-positive and Gram-negative bacteria ${ }^{(4-6)}$. However, recent microbiological 
studies have failed to provide a convincing answer to the question of which particular bacterial pathogens, if any, are capable of perpetuating chronic inflammation in the paranasal sinuses. Hypotheses regarding bacterial biofilm formation, intracellular persistence of some specific pathogens, immunological responses to Staphylococcus aureus superantigens or fungi have not accurately explained the existence of so-called "difficult-to-treat" recalcitrant CRS cases that are resistant to antimicrobial therapy (7-11).

Therefore, our prospective multicenter study was designed to investigate the spectrum of aerobic and anaerobic microorganisms inhabiting the middle nasal meatus and maxillary sinuses in CRS patients. The study also investigated resistance of the recovered organisms to various antimicrobial agents.

\section{Materials and methods}

The study was conducted during the period from May 2011 to May 2012 at four centers in Moscow (two clinics), St. Petersburg and Smolensk. All microbiological investigations were performed at the same laboratory (Institute of Antimicrobial Chemotherapy at the Smolensk State Medical Academy).

A total of 103 patients of both genders ( 48 men and 55 women) 16 to 70 years of age with CRSsNP were recruited. Diagnosis of CRS was based on EP3OS guidelines, namely: the presence of two or more symptoms, one of which was either nasal obstruction or nasal discharge (anterior/posterior nasal drip), \pm facial pain/pressure, \pm reduction or loss of smell lasting for $\geq 12$ weeks (4). Only cases with opacification of one or both maxillary sinuses on computed tomography (CT) scans performed within the last month were included in the evaluation.

Exclusion criteria were: CRSwNP, antrochoanal polyps, clinical or radiological signs of any kind of fungal maxillary sinus disease, the presence of a foreign body (dental fillings/tooth roots) in the sinus, oro-antral fistula, orbital or intracranial complications from rhinosinusitis, primary or secondary immunodeficiency (AIDS, X-linked agammaglobulinemia, common variable immunodeficiency), hereditary mucociliary clearance disorders (Kartagener and Young syndromes) and cystic fibrosis. Patients receiving systemic or topical antimicrobials within 4 weeks prior to enrollment and those with individual intolerance of topical anesthetics were also excluded. The study protocol was approved by the Interinstitutional Ethics Committee of the Sechenov First Moscow State Medical University.

\section{Sampling}

Samples for microbiological examinations were collected as follows: first, cotton pads soaked in a solution of $2 \%$ lidocaine mixed with $0.1 \%$ epinephrine were applied in the nasal cavity on the side where radiological signs of maxillary sinusitis were more prevalent (or on the side of unilateral disease). Then, a standard thin cotton probe was introduced into the middle nasal meatus with the guidance of a $0^{\circ}$ degree endoscope and placed in a test tube containing Amies transport medium with charcoal (Copan, Italy). Care was taken to avoid any contact with the walls of the nasal vestibule when inserting and removing the probe.

Secondly, a needle antral puncture was performed through the inferior nasal meatus approximately $2.5 \mathrm{~cm}$ behind the anterior head of the inferior turbinate. Then, a sterile disposable syringe was connected to the needle and $1 \mathrm{cc}$ of the sinus content was aspirated and placed in the test tube with the semi-solid ACT medium (Remel, USA) which insures the preservation and survival of both aerobic and anaerobic microorganisms for 72 hours. If the liquid content of the sinus could not be easily aspirated into the syringe, 2 cc of sterile saline was injected into the sinus and the fluid was aspirated again after 30 seconds.

\section{Culturing}

The specimens were inoculated on 5\% defibrinated horse blood agar and Columbia chocolate agar (Becton Dickinson, USA) plates for aerobic and facultative anaerobic organisms. The plates were incubated at $37^{\circ} \mathrm{C}$ in $5 \%$ carbon dioxide and examined at 24 and $48 \mathrm{~h}$. For anaerobes, the samples were placed on Wilkins-Chalgren agar (Oxoid, UK) with 5\% defibrinated horse blood and Wilkins-Chalgren agar supplemented with G-N Anaerobic Selective Supplement (Oxoid, UK), which contains growth inhibitors of Gram-positive microorganisms (nalidixic acid and vancomycin) and additional anaerobe growth promoters (haemin and menadione). The anaerobic plates were incubated in a BugBox anaerobic chamber (Ruskin Technology, UK) and examined at 48 and $96 \mathrm{~h}$. The microorganisms were identified using time-of-flight mass spectrometry (Microflex Maldi Biotyper 2.0, Bruker Daltonics, Germany).

Susceptibility to antimicrobial agents was determined using the disk diffusion method on Mueller-Hinton medium (BioRAD, France), in accordance with performance standards CLSI 2011, M100-S21 (Clinical and Laboratory Standards Institute, USA). Standard disks with antibiotics (BioRAD, France) were used. Susceptibility of S. pneumoniae was determined on MuellerHinton medium employing the same method and using disks supplemented with $5 \%$ defibrinated sheep blood. Susceptibility of S. pneumoniae to penicillin was determined by screening with a 1 mcg disk. The minimum inhibitory concentration of penicillin was checked using gradient test strips with penicillin (E-test) (Table 1) in cases where the identified areas of inhibition were less than the specified value. Susceptibility of $H$. influenzae isolates to ampicillin and amoxicillin was determined using clavulanic acid on a Haemophilus Test Medium Base, with the growth additive Haemophilus Test Medium Supplement (Oxoid, UK) (Table 1). The susceptibility of anaerobic bacteria to antibiotics was not tested because these microorganisms are still very susceptible to basic anti-anaerobic drugs, according to previous publi- 
Table 1. Antimicrobials used to determine susceptibility of various pathogens.

\begin{tabular}{|c|c|}
\hline $\begin{array}{l}\text { I Staphylococcus aureus and Stap- } \\
\text { hylococcus spp. } \\
\text { Resistance to methicillin, assessed } \\
\text { on cefoxitin disks }\end{array}$ & $\begin{array}{l}\text { Cefoxitin, } 30 \mathrm{mcg} \\
\text { Erythromycin, } 15 \mathrm{mcg} \\
\text { Clindamycin } 2 \mathrm{mcg} \\
\text { Gentamicin, } 10 \mathrm{mcg} \\
\text { Ciprofloxacin } 5 \mathrm{mcg} \\
\text { Co-trimoxazole, } 1.25 / 23.75 \mathrm{mcg}\end{array}$ \\
\hline II Enterobacteriaceae & $\begin{array}{l}\text { Ampicillin, } 10 \mathrm{mcg} \\
\text { Amoxicillin/clavulanate, 20/10 } \\
\text { mcg } \\
\text { Cefotaxime, } 30 \mathrm{mcg} \\
\text { Gentamicin, } 10 \mathrm{mcg} \\
\text { Ciprofloxacin } 5 \mathrm{mcg} \\
\text { Trimethoprim/sulfamethoxazole, } \\
1.25 / 23.75 \mathrm{mcg} \\
\text { Meropenem, } 10 \mathrm{mcg} \\
\text { Imipenem } 10 \mathrm{mcg}\end{array}$ \\
\hline $\begin{array}{l}\text { III Pseudomonas aeruginosa and } \\
\text { other non-fermenting Gram-nega- } \\
\text { tive bacteria }\end{array}$ & $\begin{array}{l}\text { Ceftazidime, } 30 \mathrm{mcg} \\
\text { Gentamicin, } 10 \mathrm{mcg} \\
\text { Amikacin, } 30 \mathrm{mcg} \\
\text { Ciprofloxacin, } 5 \mathrm{mcg} \\
\text { Meropenem, } 10 \mathrm{mcg} \\
\text { Imipenem } 10 \mathrm{mcg}\end{array}$ \\
\hline VI Stenotrophomonas maltophilia & $\begin{array}{l}\text { Trimethoprim/sulfamethoxazole, } \\
1.25 / 23.75 \mathrm{mcg} \\
\text { Levofloxacin, } 5 \mathrm{mcg}\end{array}$ \\
\hline $\begin{array}{l}\text { VII Haemophilus influenzae } \\
\text { (H.parainfluenzae) }\end{array}$ & $\begin{array}{l}\text { Ampicillin, } 10 \text { mcg } \\
\text { Amoxicillin/clavulanate, 20/10 } \\
\text { mcg }\end{array}$ \\
\hline VIII Streptococcus pneumoniae & $\begin{array}{l}\text { Oxacillin, } 1 \mathrm{mcg} \\
\text { Erythromycin, } 15 \mathrm{mcg} \\
\text { Clindamycin, } 2 \mathrm{mcg} \\
\text { Trimethoprim/sulfamethoxazole, } \\
1.25 / 23.75 \mathrm{mcg}\end{array}$ \\
\hline
\end{tabular}

Notes:

1. A $1 \mathrm{mcg}$ oxacillin disk was used to screen for the susceptibility of S.pneumoniae to penicillin.

2. Isolates of S.pneumoniae with an area of oxacillin growth inhibition $\geq 20 \mathrm{~mm}$ are assumed to be susceptible to penicillin (MIC $\leq 0.06 \mathrm{mcg} / \mathrm{ml}$ ), amoxicillin, cefuroxime, ceftriaxone, cefotaxime, ertapenem, meropenem and imipenem. The E-test was used to determine the susceptibility to penicillin of pneumococcal isolates with an area of growth inhibition $<19 \mathrm{~mm}$.

cations $^{(12,13)}$. The susceptibility of fastidious microorganisms (Streptococci and HACEK group) was not investigated for the same reason.

\section{Results}

A total of 224 samples (112 aspirates from the maxillary sinuses and 112 swabs from the middle nasal meatus) were obtained from 103 CRS patients (in 9 cases of bilateral maxillary sinusitis, specimens were taken from both sides). Forty (35.7\%) patients showed no bacteria growth in either location (maxillary sinus and middle nasal meatus). 55 (49.1\%) of maxillary sinus specimens were negative. Bacteria were isolated from the swabs taken from the middle meatus, but not from the sinus aspirates in 15 (13.4\%) cases. In contrast, 18 (16.0\%) of the patients showed microbial growth in the maxillary sinus content, but no bacteria were isolated from the middle meatus swabs.

\section{Isolates}

A total of 244 isolates representing more than 50 families were recovered, namely 164 isolates from 57 maxillary sinus cases (2.9/case) and 80 from the middle meatus cases (1.1/case). The number of isolates per specimen varied from one to seven. Altogether, 154 (63.0\%) strains of aerobic bacteria from 32 species and 90 (37.0\%) strains of anaerobic bacteria from 23 species were identified. The greatest diversity was observed among bacteria from the Streptococcus family: a total of 12 species were identified. Aerobic bacteria inhabited both the maxillary sinus cavity and the middle nasal meatus; however, the proportion of aerobic and anaerobic microorganisms was different: $55.2 \%$ vs. $44.8 \%$ in the maxillary sinus and $78.7 \%$ vs. $21.3 \%$ in the middle meatus, respectively (Figure 1).

Single isolates were identified more frequently in the middle meatus samples (39 or $63.1 \%$ of the samples), whereas microbial associations were recovered more often from the maxillary sinus aspirates (38 or $65.5 \%$ of the samples) (Figure 2).

Identical bacterial strains were isolated from the maxillary sinus and middle nasal meatus in only 10(8.9\%) cases. Among these, a single strain of S. pneumonia, $H$. influenzae, S. aureus, or $M$. morganii was recovered from 7 samples, an association of 2 isolates (S. aureus + Propionibacterium spp. and S. pneumonia + M. morganii) from two samples, and 3 different microorganisms (S. constellatus + Prevotella baroniae + Fusobacterium nucleatum) were isolated both in maxillary sinus and middle meatus in one case. Partial correlation, when the same bacteria were recovered from the middle meatus and maxillary sinus, but as a part of various microbial associations, occurred in 21 (18.8\%) patients. Among the others, S. aureus (5 samples, $4.5 \%$ ) and S. constellatus (5 samples, 4.5\%) were present in both sites more often than other organisms. The full list of microorganisms isolated from the maxillary sinus and middle meatus is presented in Table 2 .

\section{Susceptibility}

Only one microorganism, namely S. pneumoniae, which is resistant to several antibiotics (penicillin, macrolides and lincosamides) was identified in this study. A few sporadic strains of pneumococci (4 samples) with acquired resistance to macrolides were also found. All the isolates of $H$. influenza were free of penicillinases and were susceptible to the routine administration of aminopenicillins (both with and without beta-lactamase 
Figure 1. Anaerobic and aerobic microorganisms in the maxillary sinus and middle nasal meatus cultures.

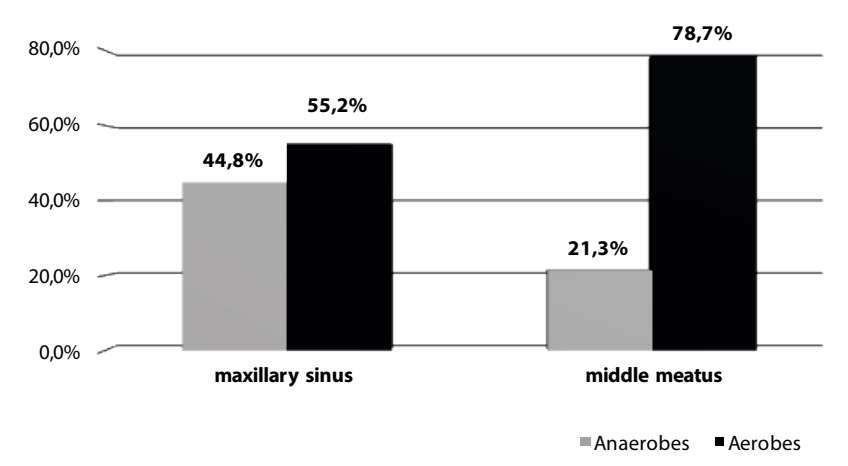

Figure 2. Single isolates and microbial associations in the maxillary sinus and middle nasal meatus cultures ( $y$-axis = number of specimens).

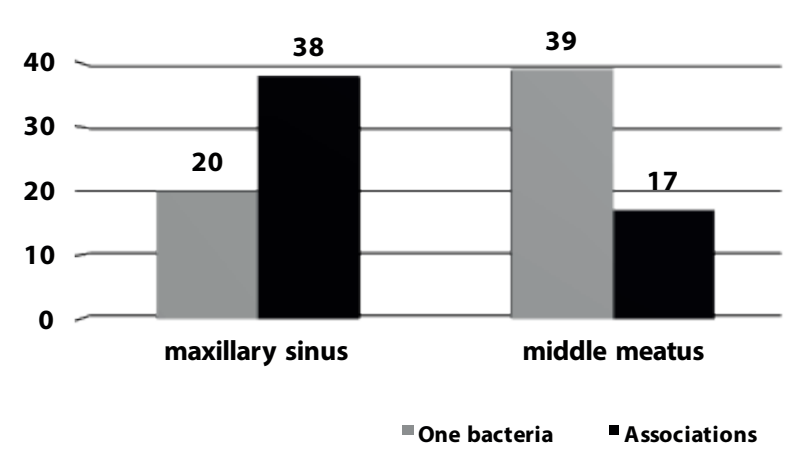

inhibitors), which is typical for Russia. Among the Enterobacteriaceae identified throughout this study, some were resistant to aminopenicillins (including beta-lactamase inhibitors), but this was a natural, not acquired resistance. Among the nonfermenting bacteria (Pseudomonas spp., Achromobacter spp. and Stenotrophomonas spp.), resistant strains were also identified, but resistance was also natural.

\section{Discussion}

The role of microorganisms in the pathogenesis of CRS, undisputed for decades, has been vigorously debated in recent years. There is no good evidence supporting the classic bacterial theory, and CRS is no longer considered to be a consequence and result of recurrent episodes of acute inflammation. The mere fact that in unilateral CRS, the same aerob ic and anaerobic bacteria can be cultured from the content of a diseased sinus and also from a healthy sinus on the opposite side casts doubt on the key role of bacteria in the etiology of the disease ${ }^{(14)}$. Recent studies have shown that complex polymicrobial communities constantly inhabit both healthy and inflamed paranasal sinus mucosa. It is important to note that the organisms residing in both healthy and diseased paranasal sinuses are often resistant to culture ${ }^{(15)}$.

It has been speculated that CRS develops when the aerobic microorganisms that are constantly present in healthy paranasal sinuses are gradually replaced by anaerobes, such as Peptostreptococcus spp., Bacteroides spp., Veillonella spp., Prevotella spp., Fusobacterium spp. Corynebacterium spp., etc., due to decreased oxygen partial pressure and a shift in $\mathrm{pH}$ into the acidic range (16,17). Unlike acute rhinosinusitis, where the "eternal trio" of upper airway pathogens (S. pneumoniae, H. influenza, and M. catharralis) plays the main role, causative pathogens of CRS have not been defined. Comparative microbiome profiling of a cohort of CRS patients and healthy subjects demonstrated reduced bacterial diversity in the CRS patients, with specific depletion of lactic acid bacteria and a relative increase in Corynebacterium tuberculostearicum ${ }^{(18)}$. In contrast, a recent study by Boase et al. has shown that the abundance of microorganisms associated with the frequent recovery of anaerobes is significantly greater in CRS patients as compared to controls. This study confirmed that S. aureus and Propionibacterium acnes were the most common organisms residing in the paranasal sinuses in CRS and healthy controls, respectively ${ }^{(15)}$.

Many previous investigations addressed CRS microbiology using conventional culture-dependent techniques. Samples were usually taken from the maxillary and frontal sinuses and the ethmoidal bulla during or after endoscopic sinus surgery. Under these conditions, the most common pathogens identified were aerobic bacteria - S. aureus ( 40\%) and coagulase-negative staphylococci $(\sim 30 \%)^{(19,20)}$.

Our study largely included CRS patients who were immediate candidates for surgical treatment, i.e., most were free of acute exacerbation. The study protocol allowed for sampling material not only from the middle nasal meatus, but also aspirates from the maxillary sinus. This means that at the time of sampling, the preceding surgical intervention did not influence the typical anaerobic conditions existing in the inflamed sinus. Our findings illustrate that streptococci (47 or $28.8 \%$ of 164 isolates) and anaerobic organisms of the Prevotella genus (29 or $17.8 \%$ of 164 isolates) were the most frequent findings in the samples from the maxillary sinuses of CRS patients.

It is well known that streptococci routinely reside mucous membranes in the nasal cavity, pharynx, gastrointestinal tract and other hollow organs under healthy conditions. Importantly, it should be noted that a true pathogen,- S. pneumoniae, was identified in only 11 (9.8\%) maxillary sinus aspirates, i.e., in 6.7\% of the total number of bacterial strains, and $23.4 \%$ of streptococci strains recovered in this study. The other 18 streptococci strains ( $16.0 \%$ of maxillary sinus aspirates, and $11.0 \%$ of the total number of isolates) belonged to the Streptococcus mitis group. These included S.oralis (7 isolates), S. mitis, S. sanguinis, S. parasanguinis, S. gordonii, S. peroris (2 isolates each) and S. infantis. 
Table 2. Microorganisms recovered from the maxillary sinus and middle nasal meatus in CRS patients.

\begin{tabular}{|c|c|c|c|c|c|}
\hline Isolates & Maxillary sinus & $\begin{array}{l}\text { Middle nasal } \\
\text { meatus }\end{array}$ & Isolates & Maxillary sinus & $\begin{array}{l}\text { Middle nasal } \\
\text { meatus }\end{array}$ \\
\hline Streptococcus constellatus & 14 & 7 & Stenotrophomas maltophilia & 1 & 0 \\
\hline Streptococcus spp. & 22 & 7 & Prevotella melaninogen. & 11 & 3 \\
\hline Enterococcus faecalis & 1 & 0 & Prevotella nigrescens & 4 & 1 \\
\hline Granulicatella adiacens & 4 & 0 & Prevotella baroniae & 4 & 1 \\
\hline Rothia muciloginosa & 2 & 1 & Prevotella bucca & 4 & 1 \\
\hline Streptococcus pneumoniae & 11 & 9 & Prevotella maculoca & 2 & 0 \\
\hline Haemopilus parainfluenzae & 2 & 0 & Prevotella spp. & 4 & 0 \\
\hline Haemophilus influenzae & 6 & 4 & Propionibacterium acnes & 4 & 3 \\
\hline Aggregatibacter aphroph. & 1 & 0 & Finegoldia magna & 2 & 1 \\
\hline Eikenella corrodens & 1 & 0 & Parvimonas micra & 7 & 2 \\
\hline Staphylococcus aureus & 11 & 17 & Fusobacterium naviforme & 5 & 0 \\
\hline Staphylococcus epidermidis & 2 & 32 & Fusobacterium nucleatum & 9 & 3 \\
\hline Citrobacter spp. & 1 & 1 & Fusobacterium vinsentii & 1 & 0 \\
\hline Klebsiella pneumoniae & 1 & 2 & Fusobacterium necrof. & 1 & 0 \\
\hline Enterobacter aerogenes & 3 & 4 & Gemella sanginis & 1 & 0 \\
\hline Enterobacter cloacae & 0 & 1 & Veillonella dispar & 2 & 0 \\
\hline Escherichia coli & 3 & 4 & Veillonella parvula & 8 & 0 \\
\hline Serratia marcescens & 0 & 1 & Slackia exigua & 1 & 1 \\
\hline Morganella morganii & 2 & 2 & Actinomyces spp. & 2 & 1 \\
\hline Pseudomonas aeruginosa & 3 & 2 & Bacteroides spp. & 1 & 0 \\
\hline Achromobacter xylosox. & 2 & 1 & Total number of isolates & 164 & 80 \\
\hline
\end{tabular}

Aggregatibacter aphrophilus (formerly Haemophilus aphrophilus) and Eikenella corrodens compose the HACEK group of microorganisms, which acquires its name from the generic names of the Gram-negative aerobic microorganisms present in the microbiome of the oral cavity. They are believed to be able to form biofilms and to cause endocarditis and other inflammatory complications. Furthermore, the growth of Granulicatella adiacens was revealed in 4 (3.6\%) maxillary sinus aspirates. These organisms, like others identified in this study, have never been reported as CRS pathogens or maxillary sinus inhabitants before. Against a prevailing background of the Prevotella microorganisms, other anaerobic bacteria, such as Peptostreptococcus spp., Bacteroides spp. and Corynebacterium spp. were quite rare.
Representatives of only two genera of anaerobic Gram-positive cocci were identified: Parvimonas micra and Finegoldia magna. It is remarkable that fungi (Candida guilliermondii) were cultured only once from the middle nasal meatus swabs and in no case from the maxillary sinus aspirates, which generally corresponds to some previous reports ${ }^{(19,20)}$.

The role of intracellular pathogens has been discussed from time to time in terms of their potential ability to contribute to the development of acute and chronic rhinosinusitis. There is limited evidence that Chlamydia and Mycoplasma can be detected in the paranasal sinuses mucosa in approximately $30 \%$ of cases ${ }^{(21)}$, but their role in the pathogenesis of CRS remains unclear. Therefore, the present study was not aimed at the identification 
of the intracellular pathogens.

Some authors explain the existence of recalcitrant forms of CRS resistant to maximal medical and surgical therapy by intracellular persistence of $S$. aureus in the mucosa of the nasal cavity and paranasal sinuses ${ }^{(9)}$. However, data on the presence of $S$. aureus in the nasal cavity and paranasal sinuses vary and largely depend on the sampling method and culturing technique. For example, one study examined middle nasal meatus microbiology and recovered S. aureus in $36 \%$ of the specimens from CRS patients, and in $39 \%$ of those from healthy controls ${ }^{(22)}$. Another study identified S. aureus in the swabs from the inferior turbinate in $50 \%$ of CRS patients and $33 \%$ of healthy individuals ${ }^{(23)}$. We were able to detect this organism in 17 (13.9\%) middle meatus swabs and 11 (9.0\%) sinus aspirates. Hence, the unique role of persistent $S$. aureus infection in the pathogenesis of CRSSNP remains doubtful and is not supported by the results of our study.

Another factor that may potentially contribute to chronification of inflammation in the paranasal sinuses is the emergence of antibiotic-resistant bacteria: It has been reported that 58-83\% of the isolates from CRS patients in the USA in 2005-2006 were lactamase-producing bacteria ${ }^{(24,25)}$. The results of our study suggest that the problem of bacterial resistance in our country is not as relevant as it is in Western Europe and America. Of the 244 strains isolated from the middle nasal meatus and maxillary sinuses in our CRS patients, there was only one with multiple resistance (S. pneumoniae), and four strains of pneumococci resistant to macrolides. The isolates of $H$. influenzae did not have penicillinases and were susceptible to conventional antibiotics used for the treatment of rhinosinusitis.

The overall results of this multicenter study allow for the conclusion that the common bacterial pathogens of acute rhinosinusitis and S. aureus seem unlikely candidates to give rise to the scenario of chronic inflammation of the paranasal sinuses mucosa. The spectrum of microorganisms residing the middle nasal meatus and maxillary sinus is extremely diverse and includes a huge number of both aerobic and anaerobic bacteria. There is an obvious tendency indicating that under conditions of chronic inflammation, anaerobic bacteria present much more frequently in the maxillary sinuses than in the nasal cavity. The other tendency illustrated by the study is that the microbial community inhabiting the maxillary sinus is more diverse than in the middle nasal meatus. Therefore, it is unlikely that any specific microorganism is responsible for the development of chronic inflamma- tion in the paranasal sinuses. Quite the opposite, a more realistic hypothesis would seem to be that chronic inflammation, driven by an immunological response to some unknown stimuli, might create conditions for persistence of organisms in the paranasal sinuses.

More studies are needed to elucidate the role of bacteria in the pathogenesis of CRS, in particular by comparing the density and composition of the microbial community residing in the nasal cavity and paranasal sinuses under conditions of acute exacerbation and the time of remission.

\section{Conclusion}

The spectrum of microorganisms inhabiting the maxillary sinus and middle nasal meatus in CRS patients is extremely diverse. It includes both typical bacterial pathogens capable of inducing acute exacerbation of rhinosinusitis (S. pneumoniae, H. influenzae and S. aureus) and many other "exotic" species, which have not been previously considered as a possible cause of CRS. In this study, it was relatively rare for common upper airways pathogens - S. pneumoniae and H. influenzae - to be cultured in the maxillary sinus aspirates ( $9.8 \%$ and $5.4 \%$, respectively). This is also true for S. aureus, which was detected in $9.8 \%$ of the maxillary sinus aspirates and $15.2 \%$ of the swabs from the middle nasal meatus. It is unlikely that these pathogens would play a major role in the pathogenesis of CRS. Increasingly, a limited number of resistant bacterial pathogens isolated from the maxillary sinus suggests that acquired bacterial resistance cannot be considered to be a significant factor causing chronic inflammation of the paranasal sinuses mucosa, at least in the Russian population of CRS patients.

\section{Authorship contribution}

OAl: collected clinical material, wrote initial manuscript; SAK: collected clinical material, reviewed the manuscript; RSK: microbiological work-up, reviewed the manuscript; OIK: microbiological work-up, reviewed the manuscript; IVO: collected clinical material, reviewed the manuscript; ONS: collected clinical material; GZP: collected clinical material, reviewed the manuscript; ASL: constructed the study design, collected clinical material, reviewed and prepared final version of the manuscript.

\section{Conflict of interest}

None

\section{References}

1. Pleis JR, Lucas JW, Ward BW. Summary health statistics for US adults: National Health Interview Survey, 2008. Vital Health Stat 10 2009; 242: 1-157.

2. Gordts F, Clement PAR, Buisseret T.
Prevalence of sinusitis signs in a non-ENT population. Otorhinolaryngology 1996; 58: 315-319.

3. Hastan D, Fokkens WJ, Bachert C, et al.Chronic rhinosinusitis in Europe - an underestimated disease. A GA2LEN study.
Allergy 2011; 66(9): 1216-1223.

4. Fokkens WJ, Lund VJ, Mullol J, Bachert C, Alobid I, Baroody F, et al. European Position Paper on Rhinosinusitis and Nasal Polyps 2012. Rhinol Suppl. 2012 (23): 1-298

5. Erkan M, Aslan T, Ozcan M, Koc N. 
Bacteriology of antrum in adults with chronic maxillary sinusitis. Laryngoscope 1994; 104(3 Pt 1): 321-24.

6. Brook I. Bacteriology of chronic sinusitis and acute exacerbation of chronic sinusitis. Arch Otolaryngol Head Neck Surg. 2006 132(10): 1099-101.

7. Prince AA, Steiger JD, Khalid AN, et al Prevalence of biofilm-forming bacteria in chronic rhinosinusitis. Am J Rhinol 2008, 22(3): 239-45.

8. Harvey RJ, Lund VJ. Biofilms and chronic rhinosinusitis: systematic review of evidence, current concepts and directions for research. Rhinology 2007; 45(1): 3-13.

9. Plouin-Gaudon I, Clement S, Huggle E, Chaponnier C, Francois P, Lew D, et al. Intracellular residency is frequently associated with recurrent Staphylococcus aureus rhinosinusitis. Rhinology 2006; 44(4): 249254.

10. Bachert C, Zhang N, Patou J, van Zele T, Gevaert P. Role of staphylococcal superantigens in upper airway disease. Curr Opin Allergy Clin Immunol 2008; 8: 34-38.

11. Ponikau JU, Sherris DA, Kern EB, et al.The diagnosis and incidence of allergic fungal sinusitis. Mayo Clin Proc. 1999; 74(9): $877-$ 884.

12. Rasmussen BA, Bush K, Tally FP. Antimicrobial resistance in anaerobes. Clin Infect Dis 1997; 24(Suppl.1): 110-120.

13. Nwaokorie FO, Ogunsola FT, Coker AO. Beta-lactamase production in anaerobic bacteria. RIF 2010; 1(3): 172-179.

14. Bhattacharyya N. Bacterial infection in chronic rhinosinusitis: A controlled paired analysis. Am J Rhinol 2005; 19(6): 544-8.

15. Boase S, Foreman A, Cleland E, et al. The microbiome of chronic rhinosinusitis: culture, molecular diagnostics and biofilm detection. BMC Infectious Diseases 2013, 13: 210 doi: 10.1186/1471-2334-13-210.

16. Kennedy DW, Thaler ER. Acute and chronic sinusitis: etiology, management, and outcomes. Infec Dis Clin Pract 1997; 6: 49-58.

17. Brook I. Bacteriology of chronic sinusitis and acute exacerbation of chronic sinusitis. Arch Otolaryngol Head Neck Surg 2006; 132(10): 1099-1101.

18. Abreu NA, Nagalingam NA, Song $Y$, et al. Sinus microbiome diversity depletion and Corynebacterium tuberculostearicum enrichment mediates rhinosinusitis. Sci Transl Med 2012; 4 (151):151ra124. doi: 10.1126/scitransImed.3003783.

19. Desrosiers M, Hussain A, Frenkiel S, Kilty S, Marsan J, Witterick I, et al. Intranasal corticosteroid use is associated with lower rates of bacterial recover in chronic rhinosinusitis. Otolaryngol Head Neck Surg 2007; 136 (4): 605-609.

20. Nigro JF, Nigro CEN, Marone SAM Voegels RL. Microbiology of the maxillary and ethmoid sinuses in patients with chronic rhinosinusitis submitted to functional endoscopic sinus surgery. Braz Otorhinolaryngol 2006; 72(2): 217-222.

21. Kapustina TA, Belova EV. [Characteristics of the course of maxillary sinusitis in children with verified chlamydia infection]. [Article in Russian]. Vestn Ross Akad Med Nauk 2013; (10): 22-5.

22. Araujo E, Dall C, Cantarelli V, Pereira A,

Mariante AR. Microbiology of middle nasal meatuses in chronic rhinosinusitis. Am J Rhinol 2003; 17(1): 9-15.

23. Kremer B, Jacobs JA, Soudijn ER, Van Der Ven AJ. Clinical value of bacteriological examinations of nasal and paranasal mucosa in patients with chronic sinusitis. Eur Arch Otorhinolaryngol 2001; 258 (5): 220-25.

24. Brook I. Discrepancies in the recovery of bacteria from multiple sinuses in acute and chronic sinusitis. J Med Microbiol 2004; 53: 879-85.

25. Busaba NY, Siegel NS, Salman SD Microbiology of chronic ethmoid sinusitis: is this a bacterial illness? Am J Otolaryngol 2004; 25(6): 379-84.

Prof. Andrey Lopatin

Medical Department

Business Administration of the President of the Russian Federation

Polyclinic No. 1

Sivtsev Vrazhek Lane 26/28

119002 Moscow

Russia

Tel. +79104000086

Fax: +7499 2411363

E-mail: lopatin.andrey@inbox.ru 